\title{
Removal of Organic Wax and Particles on Final Polished Wafer by Ozonated DI Water
}

\author{
Jae-Hwan Yi*, Seung-Ho Lee, Tae-Gon Kim, Gun-Ho Lee*, Eun-Suck Choi* and Jin-Goo Park ${ }^{\dagger}$ \\ Department of Materials Engineering, Hanyang University, Ansan 426-791, Korea \\ *Leading Technology Team, R\&D Center, Siltron Inc., Gumi 730-724, Korea
}

(Received April 29, 2008 : Accepted June 10, 2008)

\begin{abstract}
In this study, a new cleaning process with a low cost of ownership (CoO) was developed with ozonated DI water $\left(\mathrm{DIO}_{3}\right)$. An ozone concentration of $40 \mathrm{ppm}$ at room temperature was used to remove organic wax film and particles. Wax residues thicker than $200 \AA$ remained after only a commercial dewaxer treatment. $\mathrm{A} \mathrm{DIO}_{3}$ treatment in place of a dewaxer showed a low removal rate on a thick wax layer of $8000 \AA$ due to the diffusion-limited reaction of ozone. A dewaxer was combined with a $\mathrm{DIO}_{3}$ rinse to reduce the wax removal time and remove wax residue completely. Replacing DI rinse with the $\mathrm{DIO}_{3}$ rinse resulted in a surface with a contact angle of less than $5^{\circ}$, which indicates no further cleaning steps would be required. The particle removal efficiency (PRE) was further improved by combining a SC-1 cleaning step with the $\mathrm{DIO}_{3}$ rinsing process. A reduction in the process time was obtained by introducing $\mathrm{DIO}_{3}$ cleaning with a dewaxing process.
\end{abstract}

Key words $\underline{\mathrm{DIO}}_{3}$ (ozonated DI water), organic wax, dewaxer, final polished wafer, particle removal efficiency (PRE), wax removal.

\section{Introduction}

Si wafers grown by Czochralski method have been produced for device chip manufacturing through several polishing and cleaning processes. ${ }^{1)}$ As increasing the size of wafers for the mass production of device, the improved uniformity and near zero defects of Si wafers are required for the semiconductor manufacturing. In general, vacuum chuck or membrane type films are used to attach the wafers on a polisher head for polishing process. However, organic wax has been also used to attach the wafer on the head for the final batch type polishing process particularly in order to achieve the high uniformity of wafers as shown in Fig. 1. After the polishing process, organic wax and its residue should be removed from wafer back sides. A commercial and proprietary dewaxer was used to remove a thick organic wax but, several steps of SC-1 cleanings were followed for the removal of residues and particles as shown in Fig 2 (a) in a conventional wafer backside cleaning process. It requires a long process time, high temperature, much chemical and DI water waste which cause the high cost of ownership (CoO) ${ }^{2)}$ In this study, new cleaning process

\footnotetext{
Corresponding author

E-Mail : jgpark@hanyang.ac.kr (J. G. Park)
}

was developed with ozonated $\mathrm{DI}$ water $\left(\mathrm{DIO}_{3}\right)$ to achieve low $\mathrm{CoO}$ as shown in Fig. 2 (b). $\mathrm{DIO}_{3}$ is well known as a powerful oxidant for the removal of organic contaminations and it consumes no chemicals. It is also environmental friendly solution because it can be decomposed to $\mathrm{O}_{2}$ and $\mathrm{H}_{2} \mathrm{O}$ without any residue formation on surfaces. ${ }^{3-6)} \mathrm{OH}$ radicals produced by $\mathrm{O}_{3}$ and $\mathrm{H}_{2} \mathrm{O}$ were generated in the $\mathrm{DIO}_{3}$ cleaning solution. ${ }^{7}$ The ozonized anion radical $\left(\mathrm{O}_{3}{ }^{\mathrm{O}}\right)$ in ozonated solutions decomposes into an $\mathrm{OH}$ radicals immediately. ${ }^{8)} \mathrm{OH}$ radicals, which have higher redox potentials than that of ozonated DI water, remove electrons or hydrogen atoms from organic compounds and reduce them to $\mathrm{H}_{2} \mathrm{O}$. ${ }^{9)}$ In this study, $\mathrm{DIO}_{3}$ was applied to remove thick wax layer used after batch type final polishing for wafer manufacturing process. Particle removal efficiency was also investigated using $\mathrm{DIO}_{3}$ rinse instead of DI water rinse in final polished wafer cleaning.

\section{Experiments}

To perform the experiment, $200 \mathrm{~mm}$ (p-type(100)) final polished wafers and their $2 \times 2 \mathrm{~cm}$ coupon wafers coated with the organic wax (SB, Nikka-Seiko) ( 8000 $\AA$ ) at the backside were prepared. A commercial and proprietary water soluble dewaxer (Devel, Nikka-Seiko) which has a 


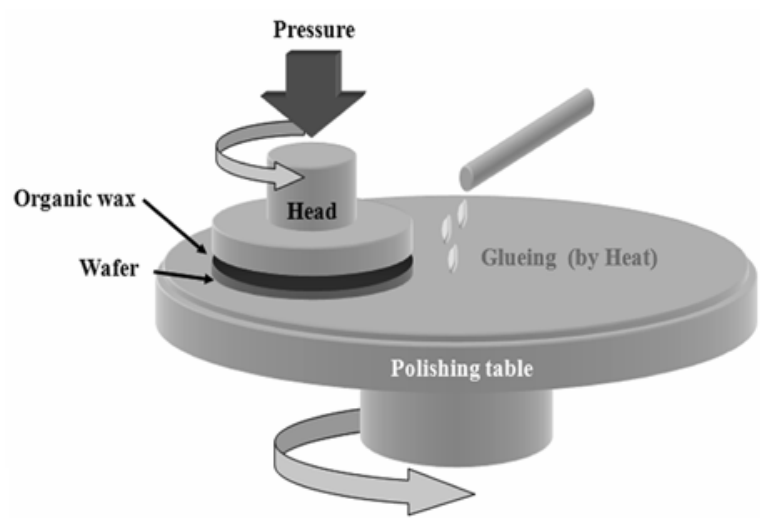

Fig. 1. Schematic diagram of the final polishing process with wax. similar chemical compounds to solvents like IPA and acetone was applied to remove the organic wax layer. The $\mathrm{DIO}_{3}$ concentration was automatically controlled by an ozone gas generation system (AX8403, MKS) with dissolved and gas ozone sensors (d-FOZZ and g-FOZZ, IN-USA) as shown in Fig. 3 by using a ozone contactor (pHasor ${ }^{\circledR}$ II, Entegris). It also could raise the $\mathrm{DIO}_{3}$ concentration by controlling the $\mathrm{O}_{3}$ gas pressure in an ozone contactor according to Henry's law. ${ }^{10-12)}$

$$
S_{l i q}=k \bullet P_{\text {gas }}
$$

$\mathrm{S}_{\text {liq }}$ is the solubility of a solute gas in the liquid, $\mathrm{P}_{\text {gas }}$ is
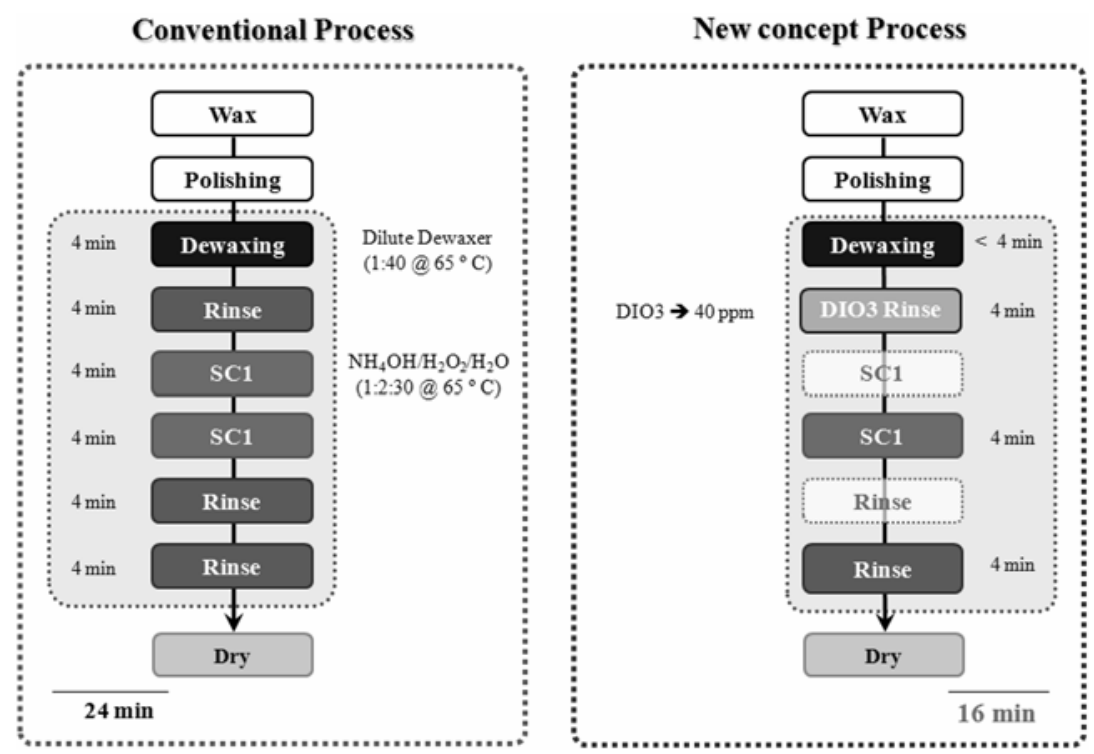

Fig. 2. Conventional cleaning process and developed cleaning procedure.

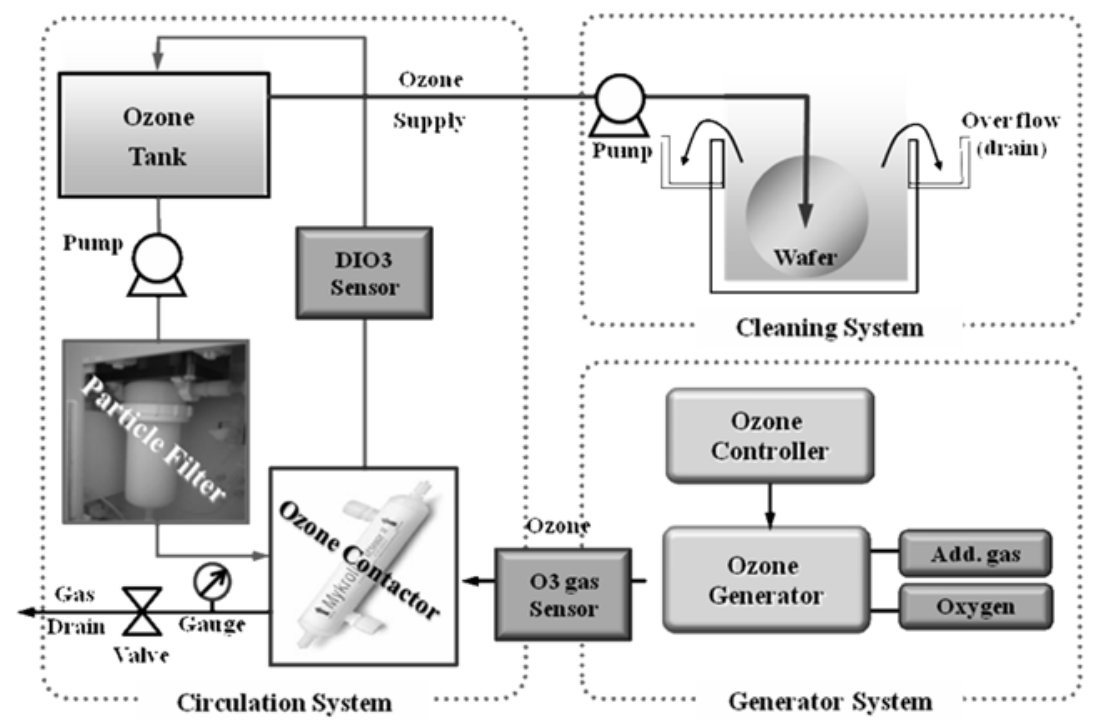

Fig. 3. Schematic diagram of the ozone cleaning system for $\mathrm{DIO}_{3}$. 
the pressure of solute gas in liquid and $k$ is Henry's constant. Fig. 4 shows the change of the dissolved ozone concentration in DI water as a function of ozone gas pressure and ozone gas concentration. The higher ozone gas pressure was more effective for higher $\mathrm{DIO}_{3}$ concentration at the same ozone gas concentration. When $\mathrm{O}_{3}$ gas pressure is too high, $\mathrm{O}_{3}$ gas could not dissolve effectively in DI water due to the excessive generation of bubbles. The optimized ozone gas pressure was used to minimize the bubble generation with a maximum dissolved ozone concentration. A reflectometer (TE-2000, $\mathrm{K}-\mathrm{MAC}$ ) was used to measure the thickness of wax films. A static contact angle analyzer (Phoenix 300, SEO) was used to observe the surface wettability. Optical microscope (LV100D, Nikon) and Fourier Transform Infrared Spectroscopy (FTX-6000, Bio-Rad) were used to analyze particles and wax residues on wafers after cleaning. Surface particle scanner (Surfscan-6200, Tencor) was used to measure the number of particles on wafer surfaces after cleanings.

\section{Results and Discussion}

\subsection{Removal of organic wax}

\subsubsection{Various solvents treatment}

A dewaxer and water soluble solvents were tested for the removal of the organic wax. The concentration of a water soluble dewaxer was changed to evaluate the removal rate of wax films. Fig. 5 shows the removal rate of organic wax as a function of time at various concentrations of dewaxer from $40: 1$ to $100: 1$ (DI water : dewaxer) ratios. A higher concentration of dewaxer was more effective in removing organic wax. Although dewaxer is effective in removing thick organic wax, it was hard to remove the organic wax completely. The wax residues thicker than $300 \AA$ were still remained after 4 minutes treatment in $40: 1$ dewaxer solution. Fig. 6 shows the change of contact angles of surfaces treated with dewaxer, IPA and acetone as a function of treatment time. IPA and acetone were as effective as a dewaxer in removing bulk wax layer. It should be noted that none of samples was reached below $20^{\circ}$ even after $5 \mathrm{~min}$ treatments.

\subsubsection{Ozonated DI water $\left(\mathrm{DIO}_{3}\right)$ treatment}

The removal of organic wax was performed in various concentrations of $\mathrm{DIO}_{3}$. Fig. 7 shows the wax removal

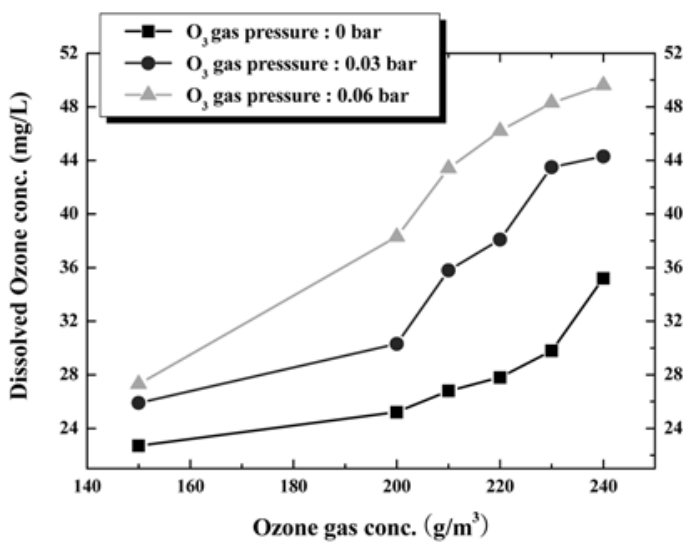

Fig. 4. Dissolved ozone concentration as functions of ozone gas pressure and concentration.

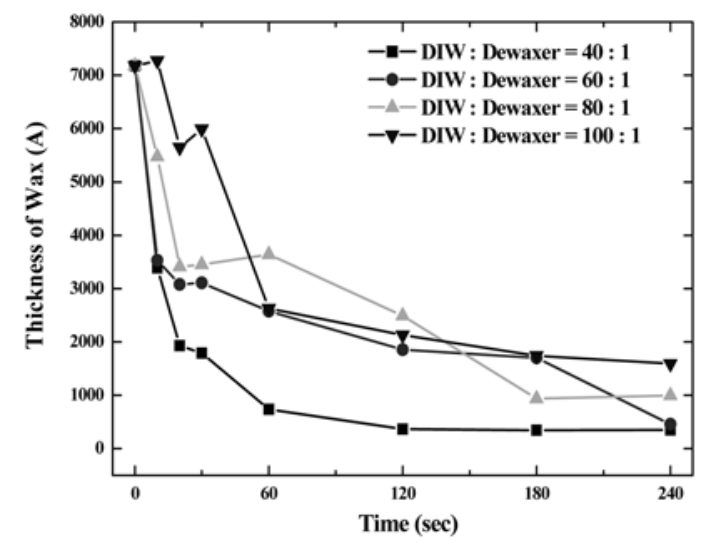

Fig. 5. Removal rate of organic wax as a function of time at various concentrations.

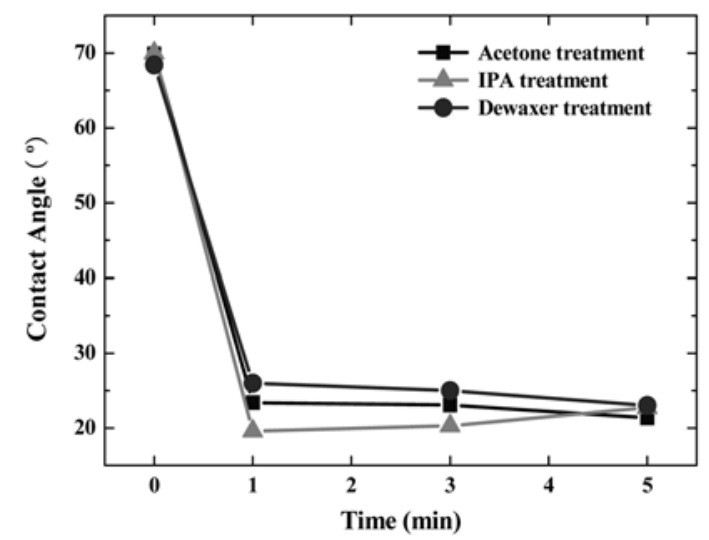

Fig. 6. Contact angles of surfaces treated with dewaxer, IPA and acetone as a function of treatment time.

rate as a function of time in both 40 and $68 \mathrm{ppm}$ of dissolved ozone concentrations. A higher concentration of $\mathrm{DIO}_{3}$ was more effective in removing organic wax. Although $\mathrm{DIO}_{3}$ is very effective in removing organic contaminants, $\mathrm{DIO}_{3}$ shows too low removal rates on 
thick wax layer. It took more than 45 min at least to reach a film thickness less than $500 \AA$ which indicates the ozone reaction is diffusion controlled process. The mechanism of organic compounds removal by $\mathrm{DIO}_{3}$ is described in Fig. 8. The reaction between hydroxide ions $\left(\mathrm{OH}^{-}\right)$which are dissolved in DI water and ozone $\left(\mathrm{O}_{3}\right)$ form superoxide anion radical $\mathrm{O}_{2}{ }^{\mathrm{o}}$ and hydroperoxyl radical $\left(\mathrm{HO}_{2}{ }^{\circ}\right)$.

$$
\begin{aligned}
& \mathrm{O}_{3}+\mathrm{OH}^{-} \rightarrow \mathrm{O}_{2}{ }^{0-}+\mathrm{HO}_{2}{ }^{0} \\
& \mathrm{O}_{3}+\mathrm{O}_{2}{ }^{0-} \rightarrow \mathrm{O}_{3}{ }^{0--}+\mathrm{O}_{2}
\end{aligned}
$$

The ozonized anion radical $\left(\mathrm{O}_{3}{ }^{\mathrm{o}}\right)$ formed by the ozone decomposes immediately into an $\mathrm{OH}$ radicals and $\mathrm{O}_{2}$ in following ways. ${ }^{13)}$

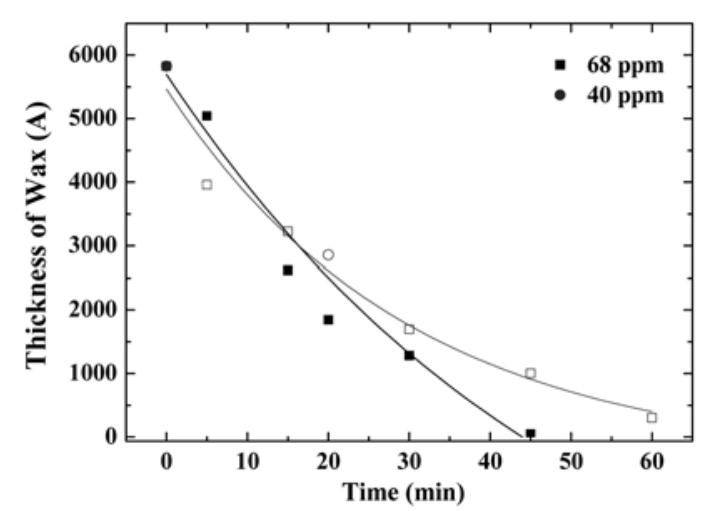

Fig. 7. Wax removal rate as a function of time in both 40 and $68 \mathrm{ppm}$ of dissolved ozone concentrations.

$$
\begin{aligned}
& \mathrm{O}_{3}{ }^{0-}+\mathrm{H}^{+} \rightarrow \mathrm{HO}_{3}{ }^{0-} \\
& \mathrm{HO}_{3}{ }^{0} \rightarrow \mathrm{OH}^{0}+\mathrm{O}_{2}
\end{aligned}
$$

Organic molecules, $\mathrm{R}$, can also act as promoters in the decay of ozone concentration. Some of them contain functional groups which react with $\mathrm{OH}$ radical and form organic radicals $\mathrm{HR}^{\mathrm{o}}{ }^{14,15)}$

$$
\mathrm{H}_{2} \mathrm{R}+\mathrm{OH}^{0} \rightarrow \mathrm{HR}^{0}+\mathrm{H}_{2} \mathrm{O}
$$

If oxygen is present, organic peroxy radical ROO can be formed. These can further react, and so enter again into the chain reaction.

$$
\begin{aligned}
& \mathrm{HR}^{0}+\mathrm{O}_{2} \rightarrow \mathrm{HRO}_{2}{ }^{0} \\
& \mathrm{HRO}_{2}{ }^{0} \rightarrow \mathrm{R}+\mathrm{HO}_{2}{ }^{0} \\
& \mathrm{HRO}_{2}{ }^{0} \rightarrow \mathrm{RO}+\mathrm{OH}^{0}
\end{aligned}
$$

In this way, $\mathrm{HRO}_{2}{ }^{\mathrm{o}}$ radical dissolves the organic compounds $\mathrm{R}$, and also oxidizes $\mathrm{RO} . \mathrm{HO}_{2}{ }^{\mathrm{o}}$ and $\mathrm{OH}^{\mathrm{o}}$ radical react again to decompose the ozone gas. The reaction rate constants for hydroxyl radicals and aromatic compounds are close to the diffusion limit. ${ }^{16)}$

\subsubsection{Combination of dewaxer and $\mathrm{DIO}_{3}$}

To achieve the removal of wax in a short time, the commercial dewaxer was combined with $\mathrm{DIO}_{3}$. The target of the new process was to reduce dewaxing time and SC-1 steps. Fig. 9 shows the change of residual wax

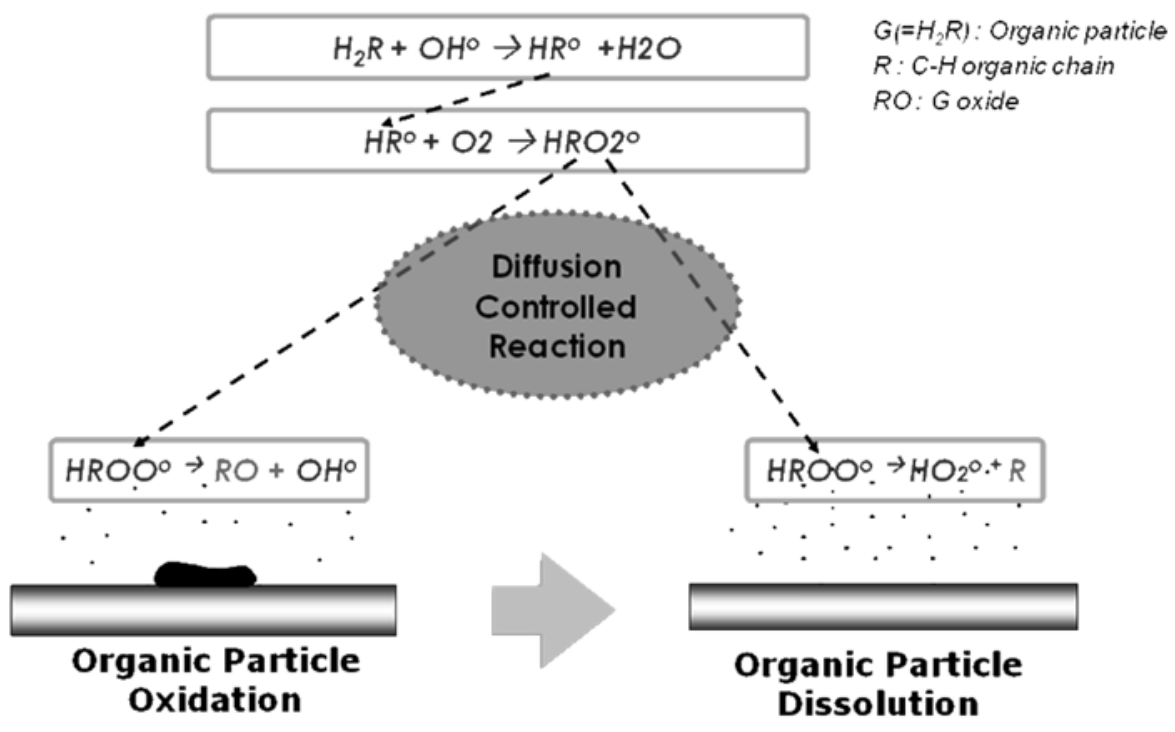

Fig. 8. Organic particle removal mechanism by $\mathrm{DIO}_{3}$ diffusion controlled reaction. 
thickness as a function of time after treating samples in a dewaxer $(1: 40)$ for $4 \mathrm{~min}$. The treatment of films in $\mathrm{DIO}_{3}$ rinsing resulted in the film thickness lower than

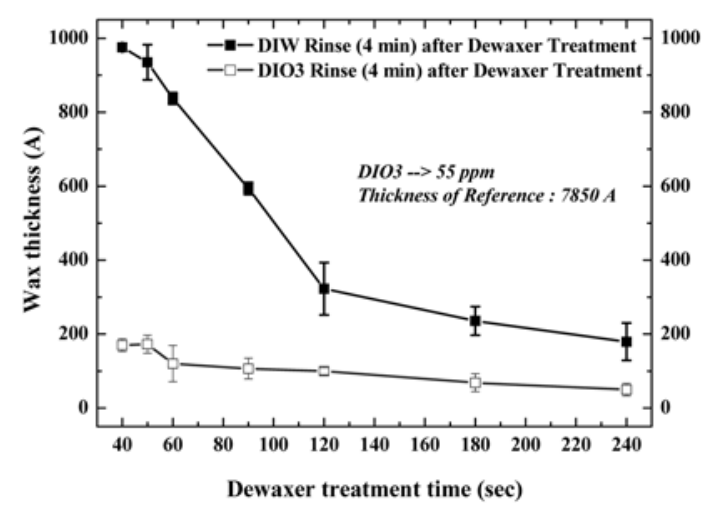

Fig. 9. Change of residual wax thickness as a function of time after treating samples in a dewaxer $(1: 40)$ for $4 \mathrm{~min}$.
$100 \AA$ even at a shorter treatment time than $1 \mathrm{~min}$. When dewaxer is treated for 4 minutes, the wax residues thicker than $200 \AA$ were still remained after conventional dewaxing and DI water rinsing process. On the other hand, $\mathrm{DIO}_{3}$ rinse instead of DI water rinse after dewaxing process had less than $50 \AA$ of wax after $4 \mathrm{~min}$ rinsing. Fig. 10 shows the contact angles and optical images of surfaces treated in $\mathrm{DIO}_{3}$. Replacing DI rinse with $\mathrm{DIO}_{3}$ resulted in lower contact angles and thinner wax residual layer as shown in Fig. 10 (b) which indicates no further cleaning steps for the removal of the wax required. When adding SC-1 step after the rinse, the contact angles become completely hydrophilic regardless of ozone treatments.

\subsection{Removal of particles}

In order to evaluate the effect of $\mathrm{DIO}_{3}$ on particle removal
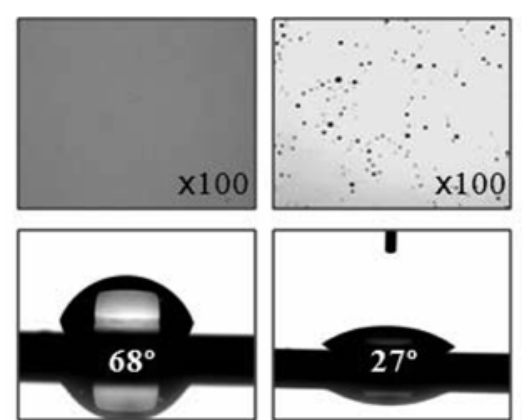

Reference

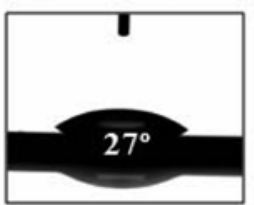

(a)

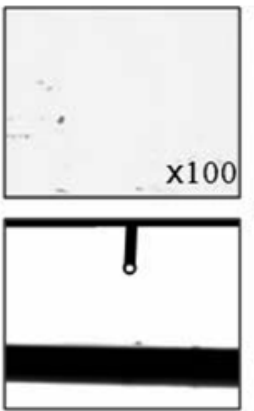

(b)

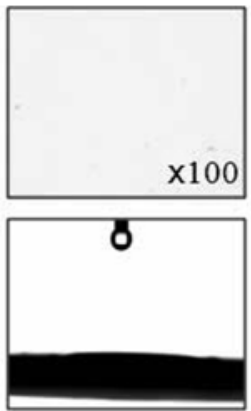

(c)

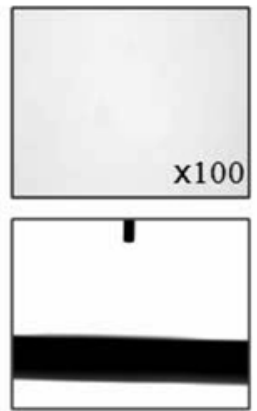

(d)

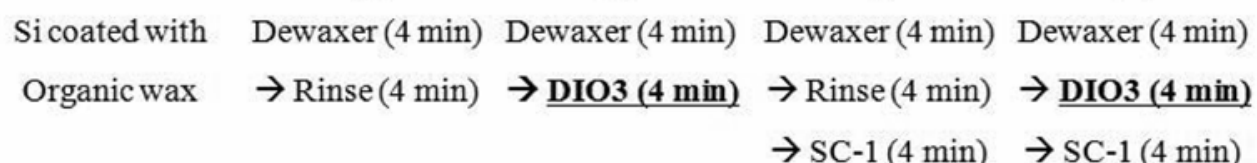

Fig. 10. Contact angles and optical images of surfaces treated in $\mathrm{DIO}_{3}$.

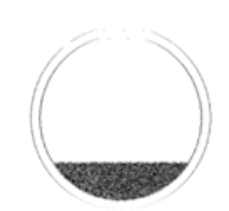

about 150000 ea

Reference

(Final polishing wafer)

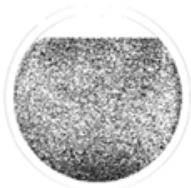

about 35000 ea

(a)

Dewaxer (4 min)

$\rightarrow$ Rinse $(4 \mathrm{~min})$

$\rightarrow$ Dry $(4 \mathrm{~min})$

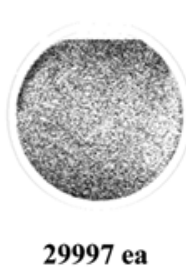

(b)

Dewaxer (4 min)

$\rightarrow \mathrm{DIO} 3$ (4 min)

$\rightarrow$ Dry (4 min)

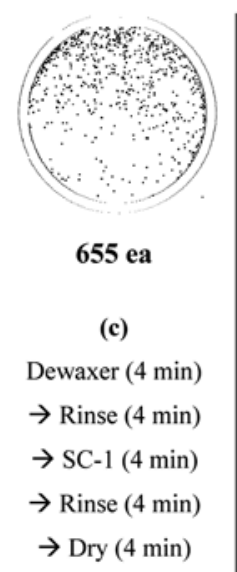

135 ea

(d)

Dewaxer (4 min)

$\rightarrow$ DIO3 (4 min)

$\rightarrow \mathrm{SC}-1$ (4 min)

$\rightarrow$ Rinse (4 min)

$\rightarrow$ Dry (4 min)

Fig. 11. Particle removal efficiency at various cleaning steps. 
efficiency (PRE), $200 \mathrm{~mm}$ p-type (100) wafers were used as shown in Fig. 11. Final drying was performed in a room temperature Marangoni type IPA dryer. $\mathrm{DIO}_{3}$ rinse after dewaxing process showed slightly lower particles than DI water as shown in Fig. 11 (b). PRE was significantly increased when SC-1 step was added in the cleaning process. It should be noted that the application of SC-1 with $\mathrm{DIO}_{3}$ showed much better PRE than with DIW rinse as shown in Fig. 11 (c) and (d) because $\mathrm{DIO}_{3}$ rinse removes not only wax residues but also particles introduced by the final polishing. PRE was almost same when compared the conventional process which has 24 minutes process time, with the developed process which has less than 8 minutes. It can also assume that $\mathrm{DIO}_{3}$ rinse makes surfaces of wafers more hydrophilic which not only prevents particles from the adhesion during rinsing but also improves the rinsing efficiency.

\section{Conclusion}

In this study, the heavy organic film, wax film used for wafer attachment on polishing head, was effectively cleaned by applying $\mathrm{DIO}_{3}$. Conventional dewaxer, IPA and acetone were used and compared one another in removing the organic wax. The wax residues thicker than $200 \AA$ were still remained after the dewaxer and solvents treatment. All of solvents decreased the surface contact angle to $20^{\circ}$ but not lower. $\mathrm{DIO}_{3}$ only did not remove the thick wax films because ozone reaction is diffusion limited. The replacement of DI water rinse with $\mathrm{DIO}_{3}$ rinse was able to completely remove not only the wax residues but also particles and reduce SC-1 clean steps. Thus the process time and chemical consumption were greatly reduced with the introduction of $\mathrm{DIO}_{3}$.

\section{Acknowledgements}

This work was funded by Siltron Inc., Korea and supported by the fostering project of the Lab of Excellency, the Research and Development program and the post BK21 program.

\section{References}

1. N. Shimoi, M. Kurokawa, A. Tanabe, N. Koizumi and Y. Matsushita, J. Cryst. Growth, 210, 31 (2000).

2. W. Kern and D. A. Puotien, R.C.A. Review, 31(2), 187 (1970). http://www.scopus.com/scopus/record/display.url?eid=2-s2.0$0014800514 \&$ view $=$ basic $\&$ origin $=$ inward $\&$ txGid $=v q$ e4qM1SXw_P8reNqhp_zhk\%3a2

3. J. Staehelin and J. Hoigene, Environ. Sci. Technol., 16, 676 (1983).

4. M. G. Alder and G. R. Hill, J. Am. Chem. Soc., 72, 1884 (1950).

5. T. Ohmi, T. Isagawa, T. Imaoka and I. Sugiyama, J. Electrochem. Soc., 139, 3336 (1992).

6. S. Noda, K. Kawase, H. Horibe, M. Kuzumoto and T. Kataoka, J. Electrochem. Soc., 152, 73 (2005).

7. J. Hoigne, H. Bader, Dis. Org. Comp., 17, 185 (1983).

8. J. Staehelin and J. Hoigne, Environ. Sci. Eng., 17, 183 (1982).

9. S. Ojima, K. Kubo, M. Kato, M. Toda and T. Ohmi, J. Electrochem. Soc., 144, 1482 (1997).

10. F. De Smedt, S. De Gendt, M. M. Heyns and C. Vinckier, Solid State Phenomena., 211, 76 (2001).

11. H. Vankerckhoven, F. D. Smedt, B. V. Herp, M. Claes, S. D. Gendt, M. M. Heyns and C. Vinckier, Ozone Sci. and Eng.,. 24, 391 (2002).

12. R. Kumar and P. Bose, Ind. and Eng. Chem. Res., 43, 1418 (2004).

13. J. Hoigne and H. Bader, Inorg. Comp. and Rad. Wat. Res., 19, 993 (1985).

14. C. V. Sonntag, J. Wat. Sup. Res. And Tech., 45, 84 (1996).

15. R. E. Buhler, J. Staehelin and J. Hoigne, J. Phys. Chem., 88, 2560 (1984).

16. G. V. Buxton, C. L. Greenstock, W. P. Helman and A. B. Ross, J. Phys. Chem., 17, 513 (1988). 